\title{
The toddler with 1 striped leg: a linear papular rash
}

\author{
Katharine Moore, Joseph M. Lam MD
}

A previously healthy 15 -month-old boy presented with a papular, erythematous rash that had developed over the previous 5 weeks. The papules were arranged in a thin vertical line that extended along the inner side of his right leg from his foot to his upper thigh (Figure 1). The patient had no other skin lesions and was not bothered by the rash. He was immunocompetent and did not have a fever or other systemic symptoms. Apart from a recent bout of viral gastroenteritis that had resolved, his medical history was unremarkable.

\section{What is your diagnosis?}

a. Inflammatory linear verrucous epidermal nevus

b. Linear lichen planus

c. Incontinentia pigmenti

d. Linear scleroderma

e. Lichen striatus

\section{How would you manage this patient's care?}

a. Parental reassurance of benign prognosis

b. Topical corticosteroids

c. Acyclovir therapy

d. Tacrolimus ointment

e. Biopsy to confirm diagnosis

See page 948 for the diagnosis and management.

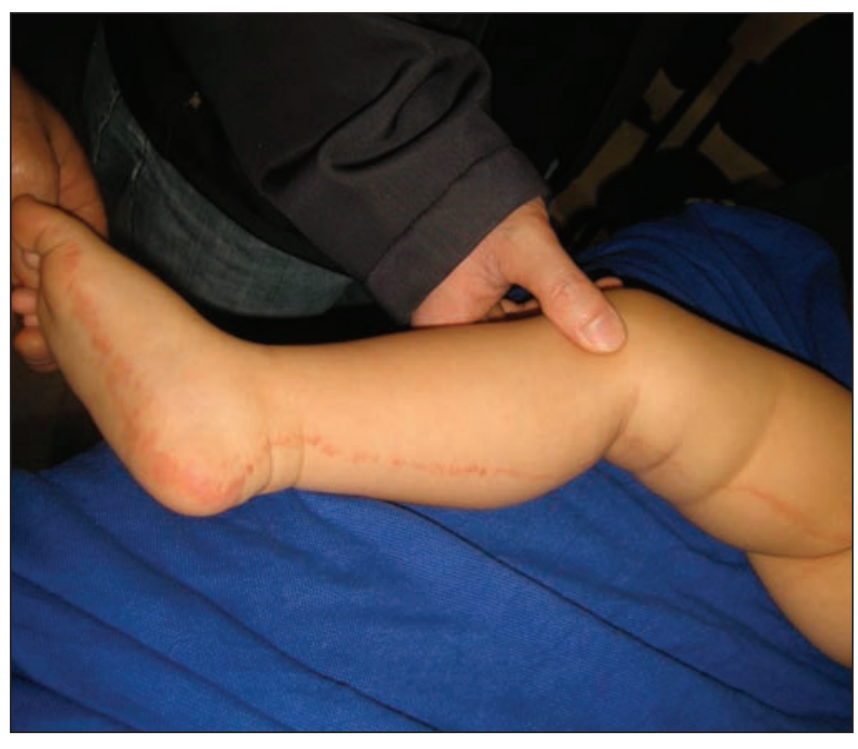

Figure 1: Photograph of the right leg of a 15-month-old boy, showing a linear, papular and erythematous rash extending from his foot to his thigh.

From the School of Medicine (Moore), University of Ottawa, Ottawa, Ont.; the Department of Pediatrics (Lam), Division of General Pediatrics, and the Department of Dermatology and Skin Science (Lam), University of British Columbia, Vancouver, BC 


\section{Discussion}

Diagnosis: The diagnosis is lichen striatus, a relatively rare, benign and self-limiting dermatosis of childhood. It is most commonly seen in children between 5 months and 15 years of age and is more common in girls than in boys. ${ }^{1,2}$ Patients usually present with sudden-onset pink or flesh-coloured, lichenoid papules that are arranged in continuous or interrupted bands. ${ }^{3}$ Occasionally, patients have hypopigmented macules and papules (classified lichen striatus albus) or involvement of the nails. ${ }^{3}$

Lichen striatus typically occurs on only 1 side of the body and affects the limbs. It can, however, occur bilaterally and on the trunk or face. ${ }^{1,4}$ In most cases, the distribution of the papules tends to follow the Blaschko lines, which are thought to correspond to the migration of embryonic skin cells. ${ }^{3}$ Although most patients have no symptoms other than the rash, some experience itchiness of the affected skin. ${ }^{1}$ The cause of lichen striatus is unknown. Potential triggers include viral infection, cutaneous injury, trauma and hypersensitivity of the skin.

Our patient's medical history and the results of a physical examination supported the diagnosis of lichen striatus. The differential diagnosis of lichen striatus includes inflammatory linear verrucous epidermal nevus, linear lichen planus, incontinentia pigmenti, blaschkitis, linear psoriasis and linear scleroderma. Inflammatory linear verrucous epidermal nevus is a unique variant of epidermal nevus. It presents as a chronic, pruritic condition with erythematous, scaly and verrucous papules that form into linear plaques. These lesions are often present at birth.

Linear lichen planus tends to be very itchy and typically occurs in adults between 30 and 60 years of age. Linear scleroderma, like lichen striatus, affects the limbs. Children with linear scleroderma, however, have joint deformities and atrophy of affected limbs. Incontinentia pigmenti is an x-linked, dominant genetic disorder and is associated with cutaneous, neurologic, ophthalmologic and dental abnormalities. The torso is the most common site of hyperpigmentation in incontinentia pigmenti, and the condition is fatal in male patients.

Management: Patients and their parents should be reassured that lichen striatus is benign and does not cause damage to the underlying muscles and tissues. They should be counselled to expect temporary hyper- or hypopigmentation of the affected skin after resolution of the papules. ${ }^{2,3}$ If lichen striatus does not resolve spontaneously within 3-12 months, the clinician should re-evaluate the diagnosis, being careful to rule out linear lichen planus and scleroderma.

The mean duration of the disease is 6 months. ${ }^{3}$ If the patient's nails are affected, complete resolution of nail deformities can take up to 30 months. Some physicians have attempted to treat lichen striatus using topical corticosteroids. Topical corticosteroid therapy may be useful to treat pruritus associated with the lesions. No difference has been found, however, in the duration of inflammatory or postinflammatory changes between patients who use topical corticosteroids and those who do not. ${ }^{3}$

Isolated cases of lichen striatus have been reported in which lesions in adult patients improved after regular treatment with tacrolimus ointment. 4.5 Whether this improvement occurred because of the tacrolimus treatment or was merely the natural resolution of the disease is difficult to determine from these reports.

This article has been peer reviewed.

Competing interests: None declared.

\section{REFERENCES}

1. Taniguchi Abagge K, Parolin Marinoni L, Giraldi S, et al. Lichen striatus: description of 89 cases in children. Pediatr Dermatol 2004;21:440-3.

2. Peramiquel L, Baselga E, Dalmau J, et al. Lichen striatus: clinical and epidemiological review of 23 cases. Eur J Pediatr 2006;165:267-9.

3. Patrizi A, Neri I, Fiorentini C, et al. Lichen striatus: clinical and laboratory features of 115 children. Pediatr Dermatol 2004;21:197-204.

4. Fujimoto N, Tajima S, Ishibashi A. Facial lichen striatus: successful treatment with tacrolimus ointment. Br J Dermatol 2003;148:587-90.

5. Sorgentini C, Allevato MA, Dahbar M, et al. Lichen striatus in an adult: successful treatment with tacrolimus. Br J Dermatol 2004;150:776-7.

CMAJ invites submissions to "What is your call?" Clinical details (including images) are presented on the first page along with a multiple-choice question about the diagnosis. The answer and a brief discussion of the condition follow on the second page. We specifically invite submissions illustrating common or important radiographic and electrocardiographic diagnoses of appeal to a general audience. We allow up to 5 references and require authors to obtain consent from the patient for publication of his or her story (form available at www .cmaj.ca/authors/checklist.shtml). Submit manuscripts online at http://mc.manuscriptcentral.com/cmaj. 